Rev. Int. Contam. Ambie. 35 (Residuos solidos en México) 29-39, 2019

DOI: 10.20937/RICA.2019.35.esp02.04

\title{
MANEJO DE LOS MEDICAMENTOS VETERINARIOS CADUCOS EN LA ZONA METROPOLITANA DEL VALLE DE MÉXICO
}

\author{
Management of veterinary expired medications in Mexico City
}

\author{
Ana Belem PIÑA GUZMÁN, Omar PRADO ROJAS, \\ María Guadalupe RAMÍREZ SOTELO y Fabián ROBLES MARTÍNEZ*
}

Sección de Estudios de Posgrado e Investigación, Unidad Profesional Interdisciplinaria de Biotecnología, Instituto Politécnico Nacional, Av. Acueducto s/n, Barrio La Laguna-Ticomán, 07340 Ciudad de México, México *Autor para correspondencia: froblesm@ipn.mx

(Recibido enero 2017; aceptado enero 2018)

Palabras clave: establecimientos veterinarios, residuos peligrosos

\section{RESUMEN}

En México, los medicamentos veterinarios caducos son considerados residuos peligrosos según la Norma Oficial Mexicana NOM-052-SEMARNAT-2005; sin embargo, un vacío en la responsabilidad de supervisión y cumplimento de la normativa origina un desorden en los procedimientos que comprenden su manejo y disposición, ya que todavía se eliminan junto con los residuos sólidos urbanos o se vierten al drenaje. Con el objetivo de conocer la situación actual del manejo de estos residuos en la Zona Metropolitana del Valle de México (ZMVM), se realizó una encuesta a 162 encargados de establecimientos veterinarios, de los cuales 112 accedieron a colaborar. El estudio reveló que más del 95\% de los encuestados está consciente de que sus establecimientos generan diversos tipos de residuos peligrosos (incluyendo medicamentos caducos) y afirmaron que algunos medicamentos veterinarios se les caducan. Aunque el $64.3 \%$ de los encuestados informó que recurren al servicio de empresas especializadas en el retiro y/o destrucción de medicamentos veterinarios caducos en la ZMVM, no existe un interés por corroborar si las mismas operan de manera legal, si dan una disposición final adecuada a los fármacos o incluso por conocer las formas de tratamiento a las que recurren, ya que el $95 \%$ de las empresas mencionadas por los encuestados no están registradas ni autorizadas por la Secretaría de Medio Ambiente y Recursos Naturales. De este estudio se concluye que si bien en México existen los mecanismos para la correcta gestión ambiental de residuos farmacéuticos, la ausencia de una normatividad específica para medicamentos veterinarios caducos hace que los métodos de manejo, tratamiento y/o disposición final sean inadecuados.

Key words: veterinary pharmacies, hazardous waste

\begin{abstract}
In Mexico, veterinary expired medications are classified as hazardous waste according to the Mexican Official Standard NOM-052-SEMARNAT-2005. Nevertheless, a void in the assignation of responsibility regarding the monitoring and fulfillment of regulations distorts the procedures comprising their managing and final disposal, since medications
\end{abstract}


are still disposed in the garbage or flushed down the toilet or sink. The aim of this study was to know the current situation of the management of these residues in the Metropolitan Zone of Mexico City (MZMC). A survey was conducted including 162 managers of veterinary pharmacies, of which 112 agreed to collaborate. Our findings revealed that more than $95 \%$ of the respondents are conscious that their establishments generate diverse types of hazardous waste (including expired medications) and claim that some veterinary medications are expired in their pharmacies. Although $64.3 \%$ of the respondents reported they use the service of companies specialized in the removal and/or destruction of expired veterinary medications in the MZMC, there does not exist an interest to corroborate if such enterprises operate in accordance to regulations, if they give a final disposition adapted to these kind of waste or even to know the management way of waste, since $95 \%$ of the companies named by the respondents are not registered nor authorized by the Secretariat of Environment and Natural Resources of Mexico. From this study, it is concluded that there are mechanisms in Mexico for the correct environmental management of pharmaceutical waste; however, the absence of specific policies for veterinary expired medications makes management, treatment and/or disposal methods inadequate.

\section{INTRODUCCIÓN}

En los últimos años, el tema de la presencia de residuos farmacéuticos en el ambiente ha despertado gran interés y preocupación por los efectos y riesgos potenciales para la salud humana y el ambiente, resultado de su inadecuado manejo y disposición final. La presencia de productos farmacéuticos en el ambiente puede tener diversos orígenes. Los pacientes (ya sean humanos o animales) se encuentran entre las fuentes importantes de contaminación, ya que los fármacos sin absorber y metabolizar y/o sus metabolitos pueden ser excretados en altos porcentajes en la orina y las heces (Elorriaga et al. 2012); o bien, una vez terminado o suspendido el tratamiento médico, los sobrantes de medicamentos, junto con los medicamentos caducos se eliminan a menudo de forma indebida siendo vertidos directamente al drenaje casero o desechados en los contenedores de residuos sólidos urbanos.

Dichos productos incluyen antibióticos, hormonas, analgésicos, tranquilizantes y productos de la quimioterapia empleados en pacientes con cáncer. Puntos importantes de generación de este tipo de residuos son los establecimientos que realizan actividades médico-asistenciales en poblaciones humanas o animales; sin embargo, no existen datos concretos sobre las cantidades generadas. Un estudio que recolectó y cuantificó los medicamentos no útiles en un hospital de tercer nivel (con varias decenas de camas) de la Ciudad de México (CDMX) durante 14 meses, encontró que el promedio mensual generado fue de $18.6 \mathrm{~kg}$ (Sánchez-González et al. 2013). Sin más detalles de estudio, estos datos no sirven para hacer una estimación de la generación de residuos de medicamentos generados en el sector salud, si se considera la amplitud de la distribución de los servicios de salud pública que cada institución de seguridad social ofrece en la Zona Metropolitana del Valle de México (ZMVM). En el campo de la asistencia médica veterinaria, la información es aún más escasa.

Los antibióticos, así como los productos con propiedades antibacterianas, antivirales o desinfectantes, son agentes generalmente muy solubles y a menudo tienen una baja biodegradabilidad, por lo que son contaminantes ambientales biológicamente activos y su manejo y disposición final inadecuados pueden generar un impacto ambiental negativo sobre ecosistemas terrestres y acuáticos (Quesada-Peñate et al. 2009, Fatta-Kassinos et al. 2011). Las prácticas inadecuadas de vertido de dichos compuestos pueden contribuir a los casos clínicos de resistencia bacteriana en animales y humanos, tanto por la exposición directa a través de agua contaminada (Amos et al. 2014) como porque favorecen su presencia en altas concentraciones en aguas domésticas residuales (Kummerer 2004). Estas últimas, por su calidad microbiológica, podrían convertirse en un gran reservorio de microorganismos portadores de genes de resistencia a antibióticos, los cuales podrían crear la resistencia antimicrobiana o antiviral en la flora humana visceral, propiciando una menor eficacia de antibióticos o productos antivirales medicinales en el futuro (Reinthaler et al. 2003). Un estudio basado en análisis metagenómico funcional demostró que la descarga del efluente de una planta de tratamiento de aguas residuales en el Reino Unido fue responsable del aumento del número de copias de genes de resistencia en el río Tamesis (Amos et al. 2014), lo que 
pone de manifiesto la ineficacia de los tratamientos presentes en las depuradoras para eliminar este tipo de contaminantes emergentes.

Se han encontrado fármacos en aguas superficiales, en aguas subterráneas e incluso en el agua potable (Quesada-Peñate et al. 2009), y gracias a los avances tecnológicos, que han mejorado la capacidad de detectar y cuantificar estos productos químicos, ahora es posible identificar sus efectos sobre la salud humana y el medio ambiente. Con métodos analíticos se ha determinado la presencia de agentes químicos a concentraciones muy bajas. Algunos estudios revelaron concentraciones de trazas de productos farmacéuticos en aguas residuales, fuentes de agua y agua potable (Fatta-Kassinos et al. 2011). Los procesos convencionales de tratamiento de agua (como la cloración) pueden eliminar aproximadamente el $50 \%$ de estos compuestos, mientras que los procesos de tratamiento más avanzados, como la ozonización, la oxidación avanzada, la nanofiltración y la ósmosis inversa, pueden lograr mayores tasas de eliminación. La ósmosis inversa, por ejemplo, puede eliminar más del 99\% de las grandes moléculas de productos farmacéuticos (OMS 2011). Aunque las concentraciones en aguas superficiales, subterráneas y parcialmente tratadas han sido inferiores a $0.1 \mu \mathrm{g} / \mathrm{l}(100 \mathrm{ng} / \mathrm{l}) \mathrm{y}$ en el agua tratada inferiores a $0.05 \mu \mathrm{g} / 1(50 \mathrm{ng} / \mathrm{l})$, estas investigaciones sugieren que los productos farmacéuticos están presentes en cuerpos de agua que reciben efluentes de aguas residuales, como los ríos, que pueden ser usados como agua de consumo para animales domésticos y ganado, así como en actividades humanas como riego de cultivos y actividades recreacionales (OMS 2011).

Aun cuando existen relativamente pocos estudios sobre el potencial tóxico de esta nueva clase de contaminantes ambientales, diferentes reportes evidencian sus efectos sobre la salud humana, así como el deterioro ecológico. De acuerdo con Philippe Garrigues, del Centro Nacional de Investigación Científica de Francia, "Los residuos de medicamentos pueden causar problemas en la reproducción de varias especies marinas y además bajan las defensas del sistema inmunitario de las especies afectadas" (BBC 2012). En 2006, la Agencia de Protección al Ambiente de Estados Unidos (US-EPA) inició un estudio piloto para investigar la presencia de productos químicos (medicamentos y productos de cuidado personal) en el tejido de peces; los resultados indicaron que los productos farmacéuticos encontrados con mayor frecuencia fueron del tipo de los antihistamínicos y antidepresivos (US-EPA 2009). Un estudio realizado por Kidd et al. (2007) evidenció los efectos de niveles altos de un estrógeno sintético (17 $\alpha$-etinilestradiol) en peces de un lago en la provincia de Ontario, Canadá. Se ha demostrado que dicho estrógeno, utilizado en las píldoras anticonceptivas, es un alterador endocrino potencial en diversas especies de vertebrados, a concentraciones del orden de ng/l. En el subcontinente indio se produjo un descenso de la población de buitres atribuido al envenenamiento por diclofenaco, un analgésico detectado en los cadáveres de los que se alimentaban estas aves (Risebrough 2004). En países como Francia, Grecia, Italia y Suecia se realizaron estudios para estimar el efecto de los efluentes y ecotoxicidad de productos farmacéuticos como carbamazepina, ácido clofíbrico y diclofenaco mediante bioensayos realizados en bacterias, algas, microcrustáceos y peces, para calcular las concentraciones predichas sin efecto (PNEC, por sus siglas en inglés) como un primer acercamiento de caracterización de riesgo (Ferrari et al. 2003).

A nivel mundial, con base en los criterios normativos de cada nación, la definición y clasificación de residuos es muy variable y no es claro si todos los residuos derivados de centros de atención a la salud de humanos y animales o investigación son peligrosos (Komilis et al. 2012). En México, el artículo 31 de la Ley General para la Prevención y Gestión Integral de los Residuos (LGPGIR) establece, en concordancia con el artículo 28 de la misma, que "Estarán sujetos a un plan de manejo los residuos peligrosos y los productos usados, caducos, retirados del comercio o que se desechen y que estén clasificados como tales en la norma oficial mexicana correspondiente", y contempla en su inciso VIII a los fármacos (SEMARNAT 2013). Además, los residuos de medicamentos (medicamentos caducos y sobrantes de medicamentos) son clasificados como residuos peligrosos según la norma oficial mexicana NOM-052-SEMARNAT-2005 (SEMARNAT 2006).

A pesar de que la legislación y normatividad mexicanas establecen que los residuos de medicamentos sean tratados como residuos peligrosos y se observen las obligaciones definidas en el reglamento de la LGPGIR, una parte importante de ellos se trata como residuos sólidos urbanos (Fig. 1) o se vierte directamente al drenaje, aun cuando el capítulo XXI de la Farmacopea de los Estados Unidos Mexicanos (FEUM), referente a la destrucción de los medicamentos caducos o deteriorados, establece que deben retirarse de los anaqueles y no deben depositarse en el drenaje, en la basura o someterse a tratamientos destructivos no autorizados; tampoco se deben tirar con su envase original, ya que podrían ser nuevamente canalizados para su venta o uso, con los consecuentes 


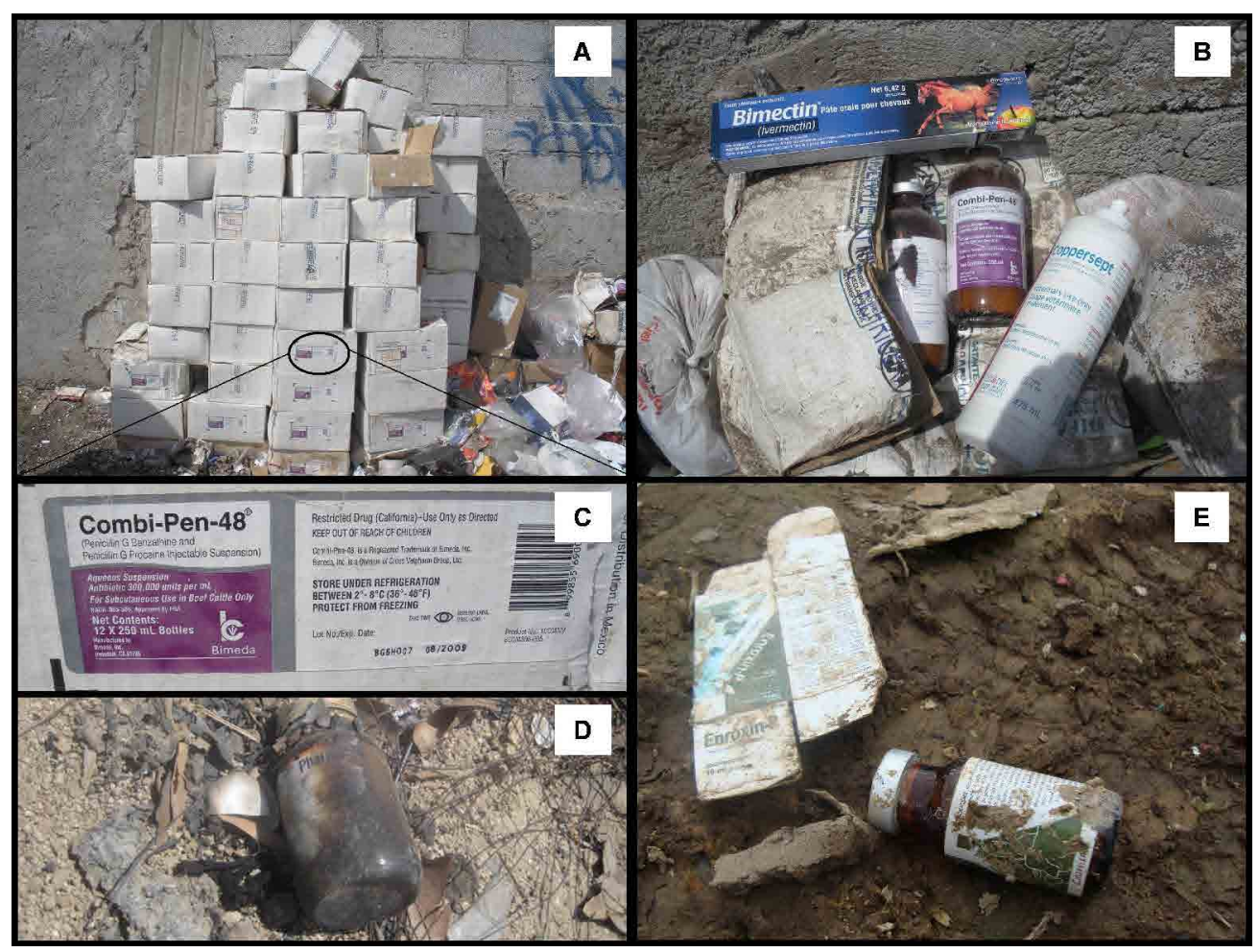

Fig. 1. Medicamentos veterinarios encontrados en sitios de disposición final de residuos sólidos urbanos. (A) Pila de cajas que contiene medicamentos veterinarios caducos; (B) una de las cajas mostradas en (A); (C) etiqueta de una de las cajas que muestra el tipo de antibióticos que contiene; (D) y (E) frascos de medicamentos encontrados en el frente de operación de dos sitios de disposición final

riesgos a la salud y al ambiente (FEUM 2010). En México se recomiendan diversos métodos de tratamiento y disposición final de residuos farmacéuticos (Hernández-Barrios et al. 1995, CENAPRED 2001); sin embargo, la Ley General de Salud y el Reglamento de Insumos para la Salud, hacen referencia a la obligatoriedad de la Farmacopea Mexicana cuando se trata de medicamentos de consumo humano, pero no para productos de uso veterinario.

Esta ambigüedad en la legislación mexicana origina fallas en el sistema de gestión y manejo integral de los residuos de medicamentos de uso veterinario, que en el sentido estricto de la palabra debieran tratarse de manera similar a los residuos de medicamentos de uso humano.

Lo anterior cobra importancia en México si consideramos que para el año 2014, Consulta Mitofsky estimó que había una mascota por cada cuatro habitantes y que el $55 \%$ de los hogares en el país tienen mascotas (generalmente perros) (Campos 2014), lo cual obedece a un mercado creciente y sostenido de mascotas (Hyun-Sook 2014). Si aplicamos estos datos al Estado de México, que cuenta con 16.64 millones de habitantes en 4.138 millones de hogares, se puede estimar que esta entidad cuenta con un aproximado de 2.27 millones de hogares con mascotas, mientras que la CDMX (con 2.646 millones de hogares y 9.06 millones de habitantes) tendría 1.45 millones de hogares con mascotas. Por lo anterior, es claro que la cantidad de establecimientos veterinarios necesarios para satisfacer la demanda de atención y medicamentos para mascotas es muy alta. Consecuentemente, se supone también alta la generación de medicamentos caducos y residuos de medicamentos en los establecimientos de atención veterinaria.

Según el Instituto Nacional de Estadística y Geografía (INEGI), en 2008 se tenían registrados 1136 establecimientos veterinarios ubicados en la CDMX (INEGI 2009), los cuales pueden generar cantidades importantes de residuos peligrosos, incluyendo medicamentos caducos. De estos establecimientos del sector privado, en dicho año 1120 brindaban servicios veterinarios para mascotas y 16 para ganadería. Evidentemente, le corresponde al médico veterinario asegurarse de que los residuos farmacéuticos generados en su establecimiento tengan una adecuada disposición final, ya que de acuerdo con la Ley Federal de Responsabilidad Ambiental (LFRA) (SEMARNAT 
2013), la responsabilidad legal por el manejo integral y la gestión de residuos peligrosos corresponde a quien los genera y podría tener implicaciones legales si sus preceptos no se cumplen.

Dado lo anterior, resulta importante conocer y dar a conocer las prácticas comunes en la gestión de medicamentos veterinarios caducos; por lo tanto, el objetivo del presente trabajo fue obtener información sobre el manejo actual de los medicamentos veterinarios caducos en los establecimientos veterinarios de la ZMVM, en particular información sobre los medicamentos de mayor demanda y el conocimiento que se tiene por parte del personal de los establecimientos veterinarios sobre tratamiento, disposición final y normatividad aplicable en el manejo de estos compuestos.

\section{METODOLOGÍA}

\section{Obtención de información}

Para el presente estudio se diseñó un cuestionario de nueve preguntas, el cual se aplicó a los propietarios y encargados de establecimientos veterinarios en la ZMVM, de enero de 2015 a mayo de 2016. Las veterinarias encuestadas se localizan específicamente en nueve delegaciones de la CDMX y 11 municipios del Estado de México, como se muestra en el cuadro I.

\section{CUADRO I. ZONA DE ESTUDIO}

\begin{tabular}{ll}
\hline Delegaciones & Municipios \\
\hline Gustavo A. Madero & Naucalpan \\
Benito Juárez & Tultitlán \\
Iztapalapa & Tlalnepantla \\
Iztacalco & Ecatepec \\
Tlalpan & Ocoyoacac \\
Coyoacán & Acolman \\
Venustiano Carranza & Cuautitlán Izcalli \\
Álvaro Obregón & Teoloyucan \\
Cuauhtémoc & Tecamachalco \\
& Nezahualcóyotl \\
& Ixtapaluca \\
\hline
\end{tabular}

\section{Aplicación de encuestas}

La encuesta diseñada contiene preguntas cerradas y abiertas con opciones de respuestas en formato binario y múltiple. La información obtenida mediante esta estrategia fue la siguente: medicamentos de mayor demanda, forma de manejo del medicamento caduco, principales industrias implicadas, nivel de conciencia, conocimiento de la normatividad vigente y los procesos de tratamiento, y disposición final de los residuos veterinarios por parte del personal que interactúa con el medicamento.

Adicionalmente, se recabaron datos como el grado de conocimiento de la normatividad vigente para el tratamiento de medicamentos caducos.

El tamaño de muestra o del número de personas (n) a encuestar que se requiere para obtener una muestra se determinó de acuerdo con la ecuación 1 (Mendenhall y Sincich 2007). Esta relación no requiere un número de población establecida, debido a que no se cuenta con un registro actualizado del número de establecimientos veterinarios vigentes en la ZMVM.

$n=\frac{Z_{\alpha}^{2} p q}{e^{2}}$

donde $n$ es el tamaño de la muestra; $Z \alpha$ el valor obtenido mediante niveles de confianza; $e$ el límite aceptable de error muestral; $p$ la proporción esperada (en este caso $5 \%=0.05$ ), variabilidad positiva; $q$ $=1-p($ en este caso $1-0.05=0.95)$, variabilidad negativa; y $e$ el límite aceptable de error muestral $(2.5 \%$ a $4 \%)$.

Se realizó la estimación entre un intervalo de confianza para una distribución normal entre 95\% y $99 \%(Z=1.96-2.58)$ en un límite aceptable de error muestral del 2.5 al 4\%. Con estos parámetros, la cantidad de encuestas que se tienen que realizar es de 105 a 114. Se aplicó la encuesta a 162 encargados de establecimientos veterinarios, de los cuales 112 respondieron. Los datos fueron analizados en Excel ${ }^{\circledR}$ v. 2007.

Para verificar la existencia de las empresas que ofrecen dar tratamiento a los residuos farmacéuticos y que fueron nombradas en las encuestas, se llevó a cabo una búsqueda en Internet y otras fuentes de información para confirmar que estuvieran registradas en la Secretaría de Medio Ambiente y Recursos Naturales (SEMARNAT). También se realizaron llamadas telefónicas para verificar los tratamientos ofrecidos por cada empresa.

\section{RESULTADOS Y DISCUSIÓN}

De un total de 162 cuestionarios aplicados, se tuvo un porcentaje de respuesta del $69.1 \%$, por lo que sólo los encargados de 112 establecimientos veterinarios de la zona de estudio se mostraron cooperativos con la encuesta. Los establecimientos veterinarios encuestados pertenecen a nueve delegaciones de la CDMX (48 encuestas) y 11 municipios del Estado de México (64 encuestas). 


\section{Empleo y caducidad de medicamentos en los es- tablecimientos veterinarios}

Los resultados de la encuesta revelaron que en la CDMX y el Estado de México los medicamentos de uso veterinario más empleados pertenecen a los siguientes grupos: vacunas, antibióticos, desparasitantes, analgésicos, antiinflamatorios, anestésicos y vitaminas, siendo las vacunas y los antibióticos los que tuvieron mayor número de menciones. Podría inferirse que al ser estos medicamentos los más utilizados, son también los que menos caducan y, por consiguiente, sus sobrantes sean mínimos; sin embargo, esto no puede aseverarse, ya que el 90.1 $\%$ de los encuestados comentaron que aun cuando tratan de ajustar sus adquisiciones a la demanda de medicamentos, sí se les llegan a caducar algunos medicamentos.

Lo anterior cobra relevancia si se considera que entre los medicamentos de mayor uso se encuentran aquellos con actividad biocida, cuyo manejo y disposición final inadecuados pueden generar impactos negativos para el ambiental y la salud. La Agencia de Administración de Medicamentos y Alimentos de Estados Unidos (US-FDA, por sus siglas en inglés) realizó una jerarquización de los antibióticos veterinarios de acuerdo con la potencial exposición humana a éstos, dividiéndolos en tres categorías: críticamente importantes, altamente importantes e importantes (US-FDA 2003). Se considerada que un agente antimicrobiano es críticamente importante cuando es el único compuesto o uno de un número limitado de compuestos disponibles para tratar una enfermedad humana grave. Por ello es indispensable garantizar el empleo prudente de dichos antibióticos tanto en humanos como en animales, ya que las bacterias resistentes a ellos pueden transmitirse a los humanos. De acuerdo con dicha clasificación, los grupos de antibióticos críticamente importantes son: cefalosporinas de tercera generación, fluoroquinolonas, macrólidos y sulfatrimetoprim.

La mayoría de los estudios que abordan la aparición de resistencia bacteriana a partir del uso de antibióticos en veterinaria, se han enfocado a explicar su origen a partir de los animales de consumo, por el uso frecuente de antibióticos con fines terapéuticos, profilácticos y como factor de crecimiento; sin embargo, teniendo en cuenta la proliferación de mascotas, el estrecho contacto con sus propietarios y el uso generalizado de antibióticos veterinarios podrían sumarse como factores asociados al origen de la resistencia bacteriana en humanos (US-FDA 2003).

Con base en los resultados de este trabajo, la enrofloxacina (el antibiótico más empleado por los encuestados) y la azitromicina pertenecen al grupo de antibióticos críticamente importantes, en tanto que la amoxicilina y la penicilina se encuentran categorizadas como antibióticos altamente importantes (US-FDA 2003). La sumatoria en la utilización de los antibióticos críticamente importantes correspondió al $10.7 \%$ del total de las formulaciones antibióticas referidas por los encuestados. Por este motivo es relevante conocer la frecuencia de desecho de este tipo medicamentos en los establecimientos veterinarios y el destino que se le da a los residuos.

\section{Empresas abastecedoras de medicamentos vete- rinarios}

Los encargados de los establecimientos encuestados nombraron 57 diferentes empresas como responsables de abastecerles medicamentos veterinarios, con un número fluctuante de menciones entre 1 y 23. Sin embargo, no todas las empresas se hacen responsables del retiro de los productos caducos. Al hacer una búsqueda en diversas fuentes, se encontró que sólo ocho de las 57 empresas mencionadas se encuentran afiliadas a la CANIFARMA (Cámara Nacional de la Industria Farmacéutica) (CANIFARMA 2012). Afortunadamente, como se muestra en el cuadro II, casi la mitad de las 11 empresas más mencionadas ( $\geq 6$ menciones) están afiliadas a dicha cámara $\mathrm{y}$, al menos en principio, tendrían la posibilidad de responsabilizarse de los medicamentos caducos abastecidos por ellas, ya que por iniciativa de dicha cámara, en 2008 se creó el Sistema Nacional de Gestión de Residuos de Envases de Medicamentos (SINGREM), cuya misión es atender los problemas generados por los medicamentos caducos. Sin embargo, la falta de interés o de conocimiento por parte del personal involucrado en el manejo de este tipo de residuos ha propiciado que, en el sector veterinario, la manera de desechar los medicamentos caducos y sobrantes de medicamentos sea inadecuada, tratándose como residuos sólidos urbanos o bien vertiéndolos en el drenaje.

CUADRO II. EMPRESAS ABASTECEDORAS DE MEDICAMENTOS VETERINARIOS EN LA CIUDAD DE MÉXICO Y MUNICIPIOS ALEDAÑOS

\begin{tabular}{ccc}
\hline $\begin{array}{c}\text { Empresas } \\
\text { referidas }\end{array}$ & $\begin{array}{c}\text { Número de } \\
\text { menciones }\end{array}$ & $\begin{array}{c}\text { Afiliación a } \\
\text { CANIFARMA }\end{array}$ \\
\hline 41 & 1 a 2 & $2 / 41(4.9 \%)$ \\
5 & 3 a 5 & $1 / 5(20 \%)$ \\
11 & $\geq 6$ & $5 / 11(45.5 \%)$ \\
\hline
\end{tabular}




\section{Destino de los medicamentos caducos generados en los establecimientos veterinarios}

En México, de acuerdo con la NOM-073SSA1-2015 (SSA 2016), un medicamento se considera caduco una vez que es rebasada su fecha de caducidad, la cual se establece con base en su formulación y características fisicoquímicas, microbiológicas y biológicas, las cuales determinan su calidad, estrictamente controlada en los laboratorios y establecimientos donde se elaboran. A pesar de ello, una vez que ha expirado la fecha de caducidad de un medicamento, o bien se han incumplido las condiciones de almacenamiento recomendadas, su buena calidad deja de garantizarse y pasan a ser un residuo. A pesar de lo anterior, un $2.6 \%$ de los encuestados sigue utilizando los medicamentos caducos (Fig. 2) argumentando que siguen teniendo el mismo efecto.

La Figura 2 muestra los diferentes destinos de los medicamentos caducos y sobrantes de medicamentos generados en los establecimientos encuestados en este estudio. Si los encuestados se dividen en dos grandes grupos, el primero de ellos (64.3\%) se constituye por la suma de todos aquellos que aseguraron que contratan a una empresa especializada en el manejo de residuos peligrosos biológicos infecciosos (RPBI) (54.5\%), y la de aquellos que acuden a depositarlos en los contenedores especiales localizados en farmacias $(9.8 \%)$, los cuales pertenecen al SINGREM, el cual garantiza su destrucción final a través de terceros autorizados de forma ambientalmente adecuada y cumpliendo con la regulación aplicable por la SEMARNAT. Este último dato es poco alentador, pues significa que sólo uno de cada 10 establecimientos recurre al servicio de dicho sistema de recolección, que fue diseñado para la gestión de residuos de medicamentos de uso humano, pero que también podría hacerse cargo de los residuos de medicamentos de uso veterinario, incluyendo envases.

Por otro lado, el segundo grupo (restante $35.7 \%$ ) incluye un porcentaje considerable de encuestados que se negaron a responder esta pregunta y los que hacen un manejo inadecuado de los residuos de medicamentos, siendo los destinos posibles la acumulación, el uso del medicamentos caducos y hasta su vertido en el drenaje o su eliminación en la basura común. La eliminación de medicamentos junto con los residuos domésticos puede derivar en su eventual incorporación al suelo y/o agua; esta problemática se agrava por el hecho de que, a nivel nacional, la forma más común de desechar los residuos urbanos es en tiraderos a cielo abierto o en sitios controlados carentes de la infraestructura necesaria para la minimización del impacto ambiental. En consecuencia, los medicamentos caducos pueden movilizarse desde la superficie hacia los mantos acuíferos por escorrentía, afectando la calidad de las aguas o bien quedar retenidos en el suelo y acumularse, con lo que se afectaría negativamente al ecosistema y a los humanos a través de las cadenas tróficas. Así, los medicamentos que son móviles en el suelo pueden ser una amenaza para el agua subterránea o lixiviar a arroyos cercanos.

De la fracción de encuestados que afirmó contratar a empresas especializadas en el manejo de RPBI, solo un poco más de la tercera parte proporcionó los nombres de 19 diferentes empresas que les brindan el servicio, mientras que un $47.2 \%$ se reservó el derecho de proporcionar el nombre de la empresa contratada. Al realizar una búsqueda en las listas oficiales de empresas dedicadas al manejo (acopio, recolección y transporte, almacenamiento e incineración) de residuos peligrosos y biológicos

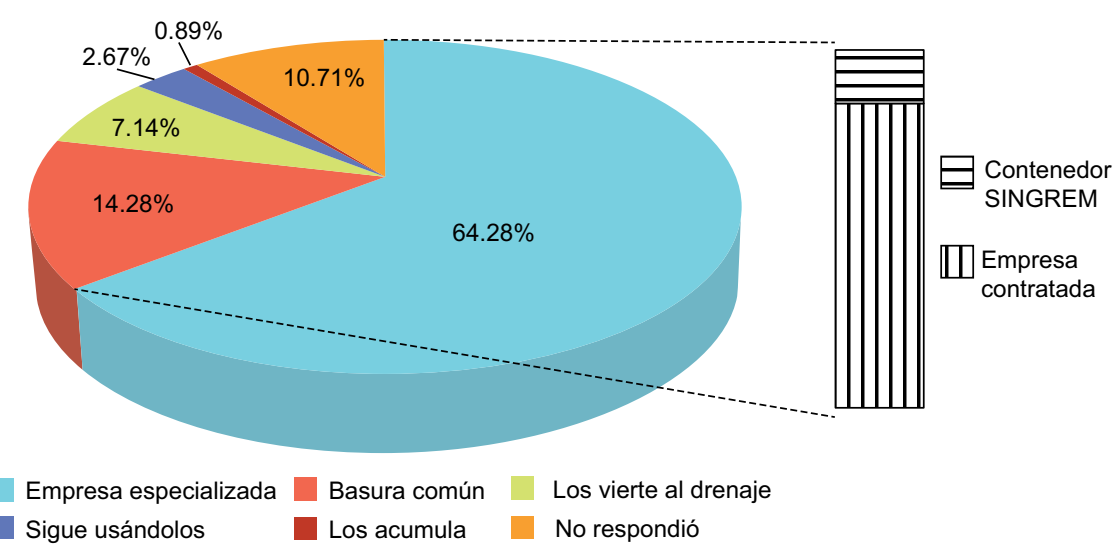

Fig. 2. Destino de los medicamentos veterinarios caducos generados en los establecimientos encuestados 
infecciosos de la República Mexicana, proporcionada por la SEMARNAT (SEMARNAT 2016), se encontró que sólo una de las empresas mencionadas (ubicada en el municipio de Lerma en el Estado de México) cuenta con registro ante dicho organismo y ante la Secretaría de Comunicaciones y Transportes (SCT), y está autorizada para llevar a cabo la incineración de RPBI y medicamentos caducos.

Paralelamente, sólo 12 de las 19 empresas mencionadas pudieron ser contactadas tras una búsqueda en Internet y otras fuentes de información, complementada con llamadas telefónicas (Fig. 3). De dichas 12 empresas, siete cuentan con equipo de incineración y sólo cuatro ofrecen este servicio (en estas últimas se incluye la empresa con autorización de la SEMARNAT y SCT), mientras que las otras tres son instituciones de educación superior y no ofrecen el servicio de incineración a particulares. Las cinco restantes son empresas cuyo giro es la distribución y venta de artículos para veterinarias. Finalmente, no se pudo constatar la existencia de siete de las 19 empresas citadas pues no cuentan con página de Internet, redes sociales u otras plataformas publicitarias. El número de establecimientos que recurren a empresas especializadas en el manejo de residuos parece alto; sin embargo, el hecho de que no se encuentre información de todas ellas podría indicar que se trata de empresas fantasma o fraudulentas, las cuales podrían realizar un mal manejo de los residuos de medicamentos, agravando así el problema de su impacto ambiental.

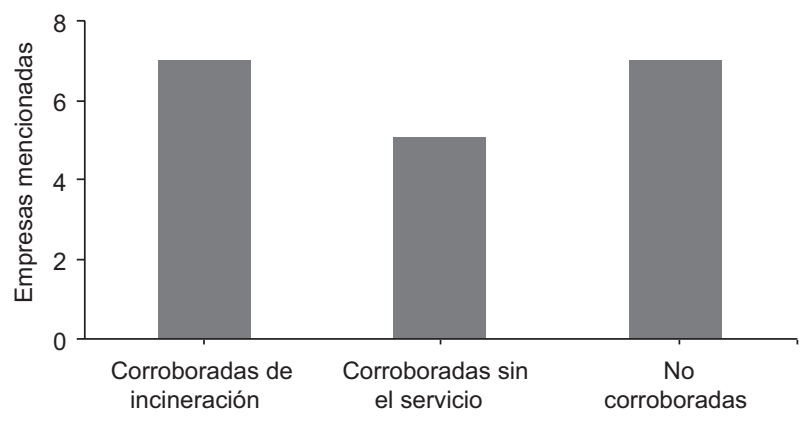

Fig. 3. Empresas especializadas en el retiro de medicamentos caducos generados en los establecimientos encuestados

En México no existe aún una Norma Oficial específica para el manejo, tratamiento y disposición final de los residuos farmacéuticos en particular, y aunque se han publicado guías para la eliminación segura de medicamentos caducos, éstas sólo son aplicables cuando los medicamentos se han acumulado en situaciones de emergencia (OMS 1999, CENAPRED 2001) y no hacen referencia a los medicamentos caducos de uso veterinario. Lo anterior no deja de sorprender, ya que desde 1989, en una guía para el manejo de residuos médicos veterinarios, se señalaba que “... los métodos más populares para su tratamiento son la esterilización por vapor (autoclave) y la incineración, y para ciertas sustancias es necesaria la descontaminación química líquida. Todos estos residuos deberían de ser embalados en contenedores resistentes a fugas o sellados, manteniendo un control mediante etiquetas de identificación" (Brody 1989). Tales métodos, además de otros más avanzados, se contemplan en el manual para el tratamiento y eliminación final de medicamentos y fármacos caducos de Hernández-Barrios et al. (1995), que es de aplicación a residuos farmacéuticos en general y por tanto debería contemplar los residuos de medicamentos veterinarios. Esto ha ocurrido recientemente en la legislación ambiental de Sudáfrica, donde los residuos de la práctica clínica veterinaria han sido incluidos en la categoría de residuos del cuidado de la salud de acuerdo con el estándar sudafricano SANS-10248 de 2004, y se reconoce a las veterinarias como generadores de residuos peligrosos y contaminantes potenciales (McLean et al. 2007), por lo que deben cumplir con sus responsabilidades sociales y ambientales.

\section{Conocimiento del marco legal en materia de re- siduos peligrosos}

Al indagar acerca del grado de concienciación de la población entrevistada se encontró que casi la totalidad $(97.3 \%)$ de los encuestados están conscientes de que en sus establecimientos veterinarios se generan residuos peligrosos, y el $87.5 \%$ de la población encuestada afirmó estar consciente del impacto negativo que el manejo y eliminación inadecuados de los medicamentos caducos provocan en la salud y el ambiente (suelo y cuerpos de agua). A pesar de ello, cerca de la mitad de los encargados de establecimientos veterinarios encuestados $(45.5 \%)$ afirmó desconocer la normatividad en materia de manejo, tratamiento y/o eliminación de medicamentos veterinarios caducos y un bajo porcentaje (6.3\%) no respondió (Fig. 4). Del $48.2 \%$ que afirmó conocer algunas normas y leyes relacionadas, sólo una fracción $(21.4 \%)$ las mencionaron, mientras que otro $26.8 \%$ que dijo conocer la normatividad correspondiente, no la mencionó. El desconocimiento de la normatividad en materia de manejo y desecho de residuos de medicamentos caducos entraña el riesgo de que éstos 
sean eliminados de manera inapropiada (vertidos al drenaje, mezclados con residuos comunes, etc.), o bien sean entregados a empresas no autorizadas para su recolección.

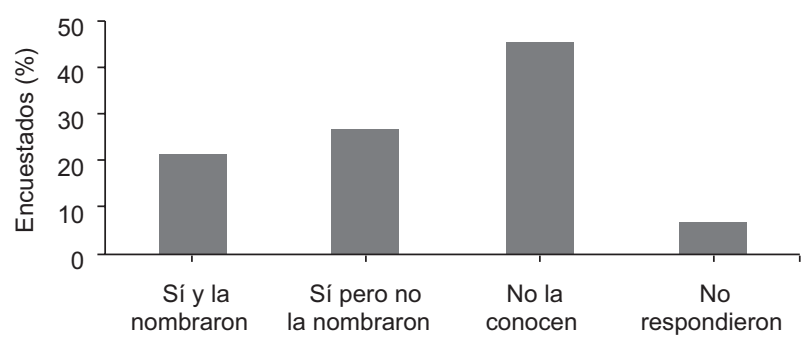

Fig. 4. Conocimiento de la normatividad en materia de residuos farmacéuticos

La información obtenida de la encuesta evidencia el nivel de conciencia del personal encargado del área de atención veterinaria con relación a la importancia del adecuado manejo de los residuos de medicamentos caducos. El grado de conocimiento de la legislación y la normatividad es un elemento determinante en el manejo de los residuos farmacéuticos, pues a pesar de que existe una conciencia de los posibles impactos negativos que éstos tendrán en el ambiente y salud, los encargados de los establecimientos de atención veterinaria no conocen con certeza la forma correcta de manejarlos. Dicha falta de información puede derivar en el comercio de estas sustancias en el mercado negro, ya que si estos residuos llegan a tiraderos o rellenos sanitarios pueden ser recuperados mediante la pepena y vendidos en establecimientos clandestinos en los que se expenden sin necesidad de receta médica. Por otra parte, existe la modalidad de venta en línea (mercado libre) de medicamentos veterinarios, sin exigencia de receta médica, incluso tratándose de antibióticos, contraviniendo así a lo especificado en el artículo 226 de la Ley General de Salud, la cual señala que "No podrán venderse medicamentos u otros insumos para la salud en puestos semifijos, módulos móviles o ambulantes" (SSA 2017).

Por lo anterior, es necesario que los generadores de residuos farmacéuticos, incluidos los establecimientos de atención veterinaria (hospitales, clínicas y consultorios) se reconozcan como generadores de residuos peligrosos e implementen un plan de manejo que contemple la destrucción o disposición adecuadas de medicamentos atendiendo a su composición química, toxicidad y estado físico, en apego a lo establecido en la normatividad. El artículo 28 de la LGPGIR (y a las obligaciones plasmadas en su reglamento) específica al manejo integral de residuos como el conjunto de actividades de reducción en la fuente, separación, reutilización, reciclaje, coprocesamiento, tratamiento biológico, químico, físico o térmico, acopio, almacenamiento, transporte y eliminación final de residuos, individualmente realizadas o combinadas de manera apropiada, para adaptarse a las condiciones y necesidades de cada lugar, cumpliendo objetivos de valorización, eficiencia sanitaria, ambiental, tecnológica, económica y social.

\section{CONCLUSIONES}

La información obtenida en este estudio evidencia que los médicos veterinarios y encargados de los establecimientos encuestados en la ZMVM saben que sus establecimientos generan residuos peligrosos y la mayoría están conscientes del impacto que éstos causan en el medio ambiente o la salud debido al manejo y eliminación inadecuados de los medicamentos veterinarios caducos; sin embargo, desconocen la existencia de normas e instrumentos legales para regular el manejo de medicamentos veterinarios caducos en México.

Aunque la normatividad y la legislación ambiental mexicanas clasifican adecuadamente los residuos farmacéuticos y los medicamentos caducos como peligrosos, no son claras en cuanto al manejo, tratamiento y disposición final de preparaciones farmacéuticas de tipo veterinario. Idealmente los medicamentos caducos y sobrantes de medicamentos deberían ponerse a disposición de empresas especializadas en el manejo y eliminación final de los mismos. Esto se cumple, en cierta medida, para los medicamentos de uso humano, cuya normatividad es clara, pero no para los de uso veterinario, donde es ambigua. Esta falta de claridad propicia que los encargados de establecimientos veterinarios adopten diversas formas de desechar los medicamentos caducos y sobrantes, de tal forma que en uno de cada cinco establecimientos veterinarios los residuos de medicamentos son desechados por el drenaje o junto con los residuos sólidos urbanos, por lo que su destino final es el relleno sanitario y pueden en ambos casos alcanzar los mantos freáticos.

A pesar de que la práctica más común reportada por los encuestados es la recolección de los residuos farmacéuticos de uso veterinario por empresas autorizadas para su destrucción y/o eliminación final, no existe un interés por corroborar si las mismas operan de manera legal y dan una destino final adecuado a estos residuos ni por conocer cuáles son las formas 
de tratamiento a las que recurren. Además, existe un vacío en la responsabilidad de supervisión y cumplimento de la normativa ambiental, entendiendo dicha responsabilidad como la competencia o capacidad para asumir, con un profundo sentido de compromiso, el deber de supervisar el cumplimiento de la ley. Dicho vacío propicia un desorden en los procedimientos que comprenden la eliminación de fármacos caducos, ya que los depósitos de residuos municipales o el drenaje persisten como formas de eliminación. Además, de acuerdo con la Ley Federal de Responsabilidad Ambiental, la responsabilidad ambiental comprende daños ambientales ya producidos, y en ese sentido los generadores de residuos de medicamentos caducos en establecimientos veterinarios tienen una responsabilidad ambiental objetiva si las acciones u omisiones relacionadas con este tipo de residuos resultan en daños ocasionados al ambiente.

Finalmente, es necesario que la industria farmacéutica y el sector gubernamental trabajen de manera conjunta en la elaboración de políticas y lineamientos aplicables a la gestión integral de los residuos peligrosos del sector veterinario, enfocándose específicamente a propietarios y empleados de establecimientos veterinarios en los que se empleen o expendan medicamentos de uso veterinario. Por otro lado, es necesario que en el sector académico se creen y refuercen líneas de investigación centradas en el desarrollo de nuevas formas de tratamiento de aguas residuales que permitan eliminar este tipo de contaminación.

\section{AGRADECIMIENTOS}

Los autores agradecen a la Secretaría de Investigación y Posgrado del IPN por el apoyo que permitió el desarrollo de este proyecto y a la Comisión de Fomento de Actividades Académicas (COFAA) del IPN por el apoyo para la presentación de los resultados en distintos foros.

\section{REFERENCIAS}

Amos G., Zhang L., Hawkey P., Gaze W. y Wellington E. (2014). Functional metagenomic analysis reveals rivers are a reservoir for diverse antibiotic resistance genes. Vet. Microbiol. 171, 441-447.

DOI: $10.1016 /$ j.vetmic.2014.02.017

BBC. (2012). Medicamentos, los nuevos contaminantes de las aguas [en línea]. https:/www.bbc.com/mundo/ noticias/2012/12/121210_ciencia_medicamentos_ oceano_dp
Brody M.D. (1989). AVMA guide for veterinary medical waste management. J. Am. Vet. Med. Assoc. 95(4), 440-52.

Campos R. (2014). México: las mascotas en nuestros hogares. Consulta Mitofsky [en línea]. http://consulta.mx/ index.php/estudios-e-investigaciones/mexico-opina/ item/577-mexico-las-mascotas-en-nuestros-hogares 01/10/2016

CANIFARMA. (2012). Cámara Nacional de la Industria Farmacéutica [en línea]. http://www.canifarma.org. $\mathrm{mx} /$ afiliados_usoveterinario.html

CENAPRED (2001). Guía para la disposición segura de medicamentos caducos acumulados en situaciones de emergencia. Centro Nacional de Prevención de Desastres, Ciudad de México, 22 pp.

Elorriaga Y., Marino D., Carriquiriborde P. y Ronco A. (2012). Contaminantes Emergentes: productos farmacéuticos en el medio ambiente. VII Congreso de Medio Ambiente. La Plata, Argentina [en línea]. http:// congresos.unlp.edu.ar/index.php/CCMA/7CCMA/ paper/view/932

Fatta-Kassinos D., Meric S., y Nikolaou A. (2011). Pharmaceutical residues in environmental waters and wastewater: Current state of knowledge and future research. Anal. Bioanal. Chem. 399, 251-275. DOI: $10.1007 / \mathrm{s} 00216-010-4300-9$

Ferrari B., Paxéus N., Lo Guidice R., Pollio A. y Garric J. (2003). Ecotoxicological impact of pharmaceuticals found in treated wastewaters: study of carbamazepine, clofibric acid, and diclofenac. Ecotox. Environ. Safe. 55, 359-370. DOI: 10.1016/S0147-6513(02)00082-9

FEUM (2010). Suplemento para establecimientos dedicados a la venta y suministro de medicamentos y demás insumos para la salud. 4a ed. Comisión Permanente de la Farmacopea de los Estados Unidos Mexicanos. Secretaría de Salud, México, 584 pp.

Hernández-Barrios C., Fernández-Villagómez G. y Sánchez-Gómez J. (1995). Manual para el tratamiento y disposición final de medicamentos y fármacos caducos. Centro Nacional de Prevención de Desastres e Instituto Nacional de Ecología, Ciudad de México, 93 pp.

Hyun-Sook L.K. (2014). El mercado creciente de las mascotas en México: beneficios y oportunidad de negocios. INCEPTUM Revista de Investigación en Ciencias de la Administración, IX (17), 7-27.

INEGI (2009). XII Censo de Servicios. Censos económicos 2009. Servicios proporcionados por el sector público. Instituto Nacional de Estadística y Geografía, México, 175 pp. [en línea]. http://www.inegi.org.mx/ est/contenidos/espanol/proyectos/censos/ce2009/ privado-paraestatal.asp 17/06/2015

Kidd K.A., Blanchfield P.J., Mills K.H., Palace V.P., Evans R.E., Lazorchak J.M. y Flick R.W. (2007). Collapse of 
a fish population after exposure to a synthetic estrogen. P. Natl. Acad. Sci. USA 174, 8897-8901.

DOI: 10.1073 /pnas.0609568104

Komilis D., Fouki A. y Papadopoulos D. (2012). Hazardous medical waste generation rates of different categories of health-care facilities. Waste Manage. 32(7), 1434-1441. doi.org/10.1016/j.wasman.2012.02.015

Kummerer K. (2004). Pharmaceuticals in the environment - Scope of the book and introduction. Pharmaceuticals in the environment. 2a. ed. Springer-Verlag, Berlín, 3-11. DOI: 10.1007/978-3-662-09259-0_1

McLean M., Watson H.K. y Muswema, A. (2007). Veterinary waste disposal: Practice and policy in Durban, South Africa (2001-2003). Waste Manage. 27(7), 902911. doi.org/10.1016/j.wasman.2006.05.004

Mendenhall W. y Sincich T. (2007). Statistics for engineering and the sciences. 5a. ed. Prentice Hall, Upper Saddle River, NJ, 1060 pp.

OMS (1999). Directrices de seguridad para la eliminación de productos farmacéuticas no deseados durante y después de una emergencia. Organización Mundial de la Salud, Ginebra, Suiza, 35 pp.

OMS (2011). Pharmaceuticals in drinking-water. Public health and environment water, sanitation, hygiene and health. Organización Mundial de la Salud, Ginebra, Suiza, 49 pp.

Quesada-Peñate I., Jáuregui H.U., Wilhelm A.M. y Delmas H. (2009). Contaminación de las aguas con productos farmacéuticos. Estrategias para enfrentar la problemática. CENIC Ciencias Biológicas 40, 173-179.

Reinthaler F.F., Posch J., Feierl G., Wüst G., Haas D., Ruckenbauer G., Mascher F. y Marth E. (2003). Antibiotic resistance of $E$. coli in sewage and sludge. Water Res. 37, 1685-1690.

DOI: $10.1016 / \mathrm{S} 0043-1354(02) 00569-9$

Risebrough R. (2004). Conservation biology: Fatal medicine for vultures. Nature 427, 596-598.

DOI: 10.1038 /nature02365

Sánchez-González E., Espinosa-Contreras C., GarcíaDomínguez J.C. y Hernández-Abad V.J. (2013). Diseño e implementación de un programa de recolección y clasificación sistemática de medicamentos no útiles en un hospital de tercer nivel. Rev. Mex. Cienc. Farm. 44 (2), 46-54.

SEMARNAT (2003). "Ley General para la Prevención y Gestión Integral de los Residuos". Secretaría de Medio Ambiente, Recursos Naturales y Pesca. Diario Oficial de la Federación, México, 8 de octubre.

SEMARNAT (2006). Norma Oficial Mexicana NOM052-SEMARNAT. Que establece las características, el procedimiento de identificación, clasificación y los listados de los residuos peligrosos. Secretaría de Medio Ambiente, Recursos Naturales y Pesca. Diario Oficial de la Federación, México, 23 de junio.

SEMARNAT (2013). Ley Federal de Responsabilidad Ambiental. Secretaría de Medio Ambiente, Recursos Naturales y Pesca. Diario Oficial de la Federación, México, 7 de junio.

SEMARNAT (2016). Empresas autorizadas. Trámites SEMARNAT [en línea]. https://www.gob.mx/ semarnat/documentos/empresas-autorizadas-para-elmanejo-de-residuos-peligrosos 04/12/17

SSA (2016). Norma Oficial Mexicana NOM-073SSA1-2015. Estabilidad de fármacos y medicamentos, así como de remedios herbolarios. Secretaría de Salud. Diario Oficial de la Federación, México, 7 de junio.

SSA (2017). Ley General de Salud. Últimas modificaciones a la nueva ley publicada el 7 de febrero de 1984 . Secretaría de Salud. Diario Oficial de la Federación, México, 22 de junio.

US-EPA (2009). Pilot study of pharmaceuticals and personal care products in fish tissue [en línea]. https:// www.epa.gov/fish-tech/pilot-study-pharmaceuticalsand-personal-care-products-fish-tissue

US-FDA (2003). Guidance for industry: Evaluating the safety of antimicrobial new animal drugs with regard to their microbiological effects on bacteria of human health concern. FDA Guidance 152. United States Food and Drug Administration, Center for Veterinary Medicine, Rockvile, MD, 35 pp. 